\title{
Cloud Computing: A review of the Concepts and Deployment Models
}

\author{
Tinankoria Diaby \\ School of Technology, Asia Pacific University of Technology and Innovation (APU), Kuala Lumpur, Malaysia \\ E-mail: diabytinankoria@gmail.com \\ Babak Bashari Rad \\ School of Computing, Asia Pacific University of Technology and Innovation (APU), Kuala Lumpur, Malaysia \\ E-mail: babak.basharirad@apu.edu.my
}

\begin{abstract}
This paper presents a selected short review on Cloud Computing by explaining its evolution, history, and definition of cloud computing. Cloud computing is not a brand-new technology, but today it is one of the most emerging technology due to its powerful and important force of change the manner data and services are managed. This paper does not only contain the evolution, history, and definition of cloud computing, but it also presents the characteristics, the service models, deployment models and roots of the cloud.
\end{abstract}

Index Terms - Cloud Computing; The Evolution, History, and Definition of Cloud Computing; Characteristics of Cloud Computing; Cloud Computing Service Models; Cloud Computing Deployment Models; Roots of the Cloud.

\section{INTRODUCTION}

In today world, every institution needs to start searching out where exactly Cloud Computing (CC) is required in their business so that they will gain a competitive advantage by staying and remaining competitive in their business sector. An exceptional characteristic of cloud computing is it pay per use one as the cloud user is only required to pay just for the used services [1].

Briefly, this paper presents a comprehensive analysis of the cloud computing, explaining its services and deployment models, and identifying various characteristics of concern.

This paper provides a literature review on concepts and deployment models of cloud computing. The structure of this paper is organized as follows. After the introduction, the evolution, history, and definition of cloud computing will be given in next section. Then, the essentials characteristics of cloud computing will be briefly explained. Service models of cloud computing will be discussed in next section, and then Cloud computing deployment models will be reviewed. The last section of the paper contains a discussion of the roots of cloud computing, then a conclusion, and finally, the references of the paper will be listed.

\section{The Evolution, History AND Definition of Cloud COMPUTING}

Cloud computing is not exactly a new technology concept as it seems to be originated after the computer diagrams network that represents the internet like a cloud [2]. The emerging technology has been very significant in both business environment and academic environment [3] Many definitions have been given to it in different ways and the researcher has noticed that all these tons of definitions mainly focused on the service and technical characteristics. For the past few years, the most used definitions among all the attributed definition to the $\mathrm{CC}$ remain the NIST definition, which stands for the National Institute of Standards and Technology [4]. According to NIST on the definition of CC, it is a model which enable suitable, on-demand access network to distribute band of configured computing assets such as network, storages, servers, services and application which precipitously provisioned and released with minimum management effort or interaction provider cloud [5]. In the other hand, another author said that $\mathrm{CC}$ is a set of applications, hardware and system software aimed to deliver good quality of services (QoS) to the end user throughout the used of the internet [6]. According to Böhm et al. [7], cloud computing is an innovation that can be seen in different ways, particularly from the technology perspective which happens to be an advancement computing as well as applying virtualization concepts to be able to utilize hardware more effectively and efficiently. He furthermore explained that cloud computing can possibly change the way, how computing resources and applications are cloud computing and computing evolution provided, breaking up traditional value chains and making room for new business models. Cloud Computing cannot be given a general definition because of the fact that its application is wide, therefore, the definitions are dependent on what integration it would be used for.

Böhm et al. [7] defined it also as an IT deployment model that is based on virtualization in which the related resources such as infrastructure, applications and data are been deployed over the internet as a form of distributed service by the service provider responsible for providing 
that service. The service can also be scaled based on individual demands, as the pricing can be flexible to the extent that it can be on a pay-per-use basis. Another definition given by Stavinoha [8] says that cloud computing is a model that can be used to enable convenient network access based on demand to a shared pool of computing resources that is configurable (for example, networks, servers, storage, applications, and services) and can quickly provisioned to be released with the most minimum management and effort from the service provider as well as their respective interaction. For Rashmi et al. [9] CC can be defined looking at two (2) viewpoints such as the user and organization viewpoints. Therefore, for the user viewpoint, CC delivers a significant for obtaining computing based services without the need of deeply knowing the fundamental technology used, and for the organization it offers services for the consumers and the business need in the easiest manner by delivering unbounded scale and differentiated service quality to foster speedy innovation and making decision. The concept of CC refers to a system where the resources of a data centre are shared by using virtualized technology that can also deliver elastic, on-demand and instant services to customers and let the customer pay by using the pay per use method [10]. This definition is graphically depicted in Fig. 1. However, according to Oliveira et al. [11], even though CC is not totally a new concept, $\mathrm{CC}$ faced a lake of standardized definition.

Different definitions from different researcher showing the strength of $\mathrm{CC}$ a different perspective but all centred around one thing: in their respective definition, they all say it is one form of the model. This makes it very distinct and more emphasis is laid on the model itself. Before the models are considered, there is a need to examine the characteristics of cloud computing critically, as it deals and relates to the models.

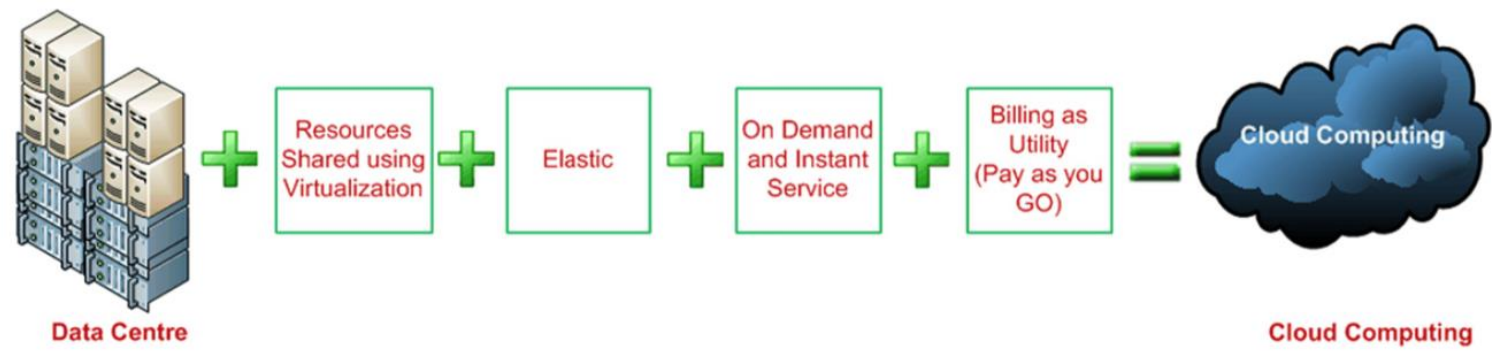

Fig.1. The schematic definition of cloud computing [10].

\section{The EsSENTIAL CHARACTERISTICS OF CLOUD COMPUTING}

Essential characteristics of cloud computing as explained by different researchers including Dillon et al. [12], Mell \& Grance [5], Srinivas et al. [13], and Stavinoha [8]. According to the NIST definition of cloud computing, essential characteristics of cloud computing are the following five characteristics:

\section{A. The on-demand self-service}

This is explained in terms of users, which can unilaterally provide computing capabilities that is needed automatically without the supervision or interaction of a human from each service provider. The computing capabilities can be server time or network storage

\section{B. The broad network access}

This is explained using computing capabilities that are available via the internet or network and can be accessed through a channeled and standard mechanism, which is put in place to promote the use of heterogeneous platforms, which can be either very thin or very thick. Examples of the platforms might include smartphones, tablets, laptops, and workstation computers.

\section{The resource pooling}

The computing resources of the provider are pooled to serve multiple users using a multi-tenant model, with different physical and virtual resources dynamically assigned and reassigned according to consumer demand. There is a sense of location-independence in that the customer generally has no control or knowledge over the exact location of the provided resources but may be able to specify location at a higher level of abstraction (e.g., country, state, or data centre)

\section{The rapid elasticity}

In this case, computing capabilities are explained in terms of the elasticity that is provided as well as released. The release might be automatic in some cases in order to actually scale inwardly and outwardly. This scaling is also used to commensurate the demand from customers. From the user's perspective, the capabilities that are made available often appears as if it is unlimited which can be appropriated in terms of quantity and time.

\section{E. The measure service}

Cloud system, in this case, is controlled and optimised automatically with the resource that is used by the leverage: this is a metering capability that is used as abstraction at some point as it is seen appropriate to the 
exact type of service. The resource can also be used to do a lot more like monitoring, controlling, and also reporting as it further provides transparency for the provider and user as well as far as utilised service are concerned.
Examples of service, in this case, includes storage, processing, bandwidth, and active user accounts.

Fig. 2 briefly demonstrates the essential characteristics of cloud computing.

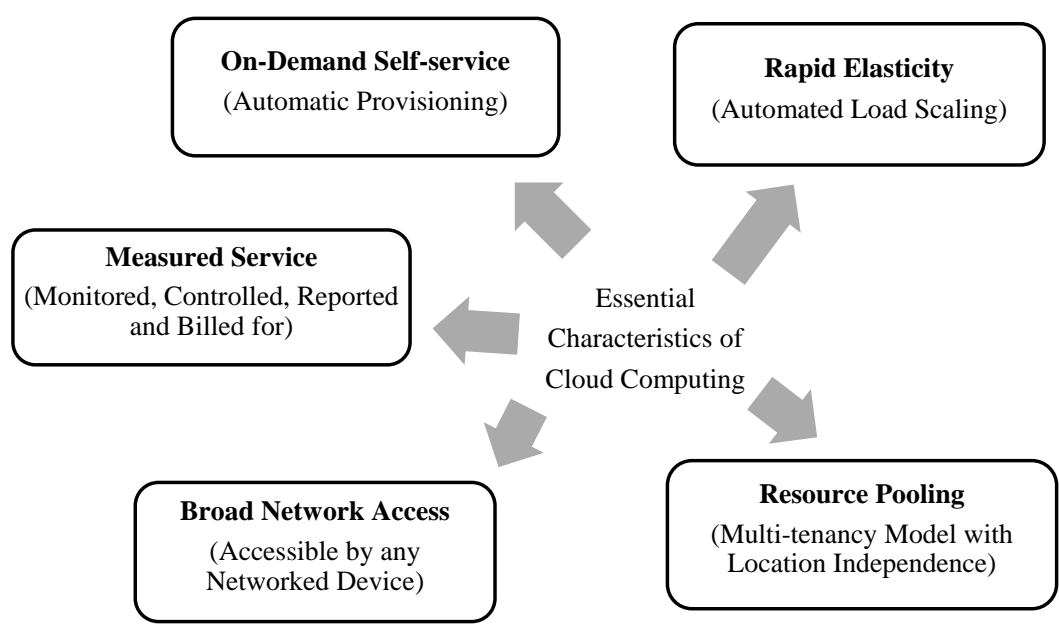

Fig.2. The Essential Characteristics of Cloud Computing.

\section{THE SERVICE MOdels OF ClOUd COMPUTING}

According to the NIST CC consists of three (3) principal model services which the Software as a Service (SaaS), the Platform as a Services (PaaS) and the Infrastructure as a Service (IaaS) [5].

The service models of CC are made based on modernday data centres which integrate the three (3) service models which are the Software as a Service (SaaS), Platform as a Services (PaaS) and Infrastructure as a Service (IaaS) and provide them as utilities by letting consumers to pay just for what they use (pay per use.) Data centres provide the hardware in which the clouds run on and they form the foundation of the cloud. Data centres are generally built of numerous servers linked with each other; and are sited in thickly crowded bands, where there is minimal risk of a natural disaster [1].

\section{A. The Software as a Service (SaaS)}

Software as a Services or Software- as a Products well known as (SaaS) is the first layer of CC service models is the platform which enables various users at the same time via the used of object code and data [14]. It is different from the traditional software as it needs own traditional software and hardware which SaaS does not need [15]. SaaS software is bought and installed into a personal computer, like a model of distribution where applications are accessible by vendors and providers of services, and provide the availability of the data to the end users via a typical platform mostly the internet. It is appropriate a progressively predominant distribution model since it underlined the technology that carries service-oriented architecture (SOA) and web services advanced and innovative developing methodologies start to become famous. Software as a Service is moreover frequently related to a licensing model such as pay-as-you-go subscription. Additionally, service broadband has been progressively accessible to sustenance end user to have access to more regions all over the globe [16]. According to the above statement, the best example will be Google Docs.

\section{B. The Platform as a Service (PaaS)}

Platform as a Service (SaaS) model is a middleware of CC service models which offers a platform of computing and stack solution like a service [17]. This model allows user or customers to build their own using software providers' libraries or tools and maintain the deployment of the software and other services. the cost of this model is reduced to businesses, as they don't necessity to be managed both software and hardware needed to create the application, meaning that Platform as a Service (PaaS) model provides applications deployment by lowered the expenses and complication of purchasing and controlling both hardware and software and provisioning capabilities of hosting [18]. The example of Platform as a Service (PaaS) examples according to the given explanation will be SQL database, Microsoft's Azure.

\section{The Infrastructure as a Service (IaaS)}

Infrastructure as a Service (IaaS) is the third service model of $\mathrm{CC}$ and it is the most comprehensive. In the IaaS, the supplier provisions the needed processing, networks storage, and additionally necessary resources of computing and the customers are allowed to implement and run many sorts of software that might be needed such as operating applications and systems. The customers do not administer or maintain the underlined $\mathrm{CC}$ system but have total power over the systems operating such as space, applications implemented, and perhaps regulator that is limited for networking selection components [3]. This model provides platform as computer environment or infrastructure (both hardware and software) for the users. 
The service model is payment scheme is defined based on the usage meaning that user only pays only for the service the user has used the billing payment can be based on the amount of storage per GB like the internet mobile data used in GB, data transfer, usage of computing per hour
[19]. The suitable example of Infrastructure as a Service (IaaS) based on the above statements is host firewalls but beside that another examples of the IaaS is the Amazon's Web Services Elastic Compute Cloud (EC2) and Secure Storage Service (S3).

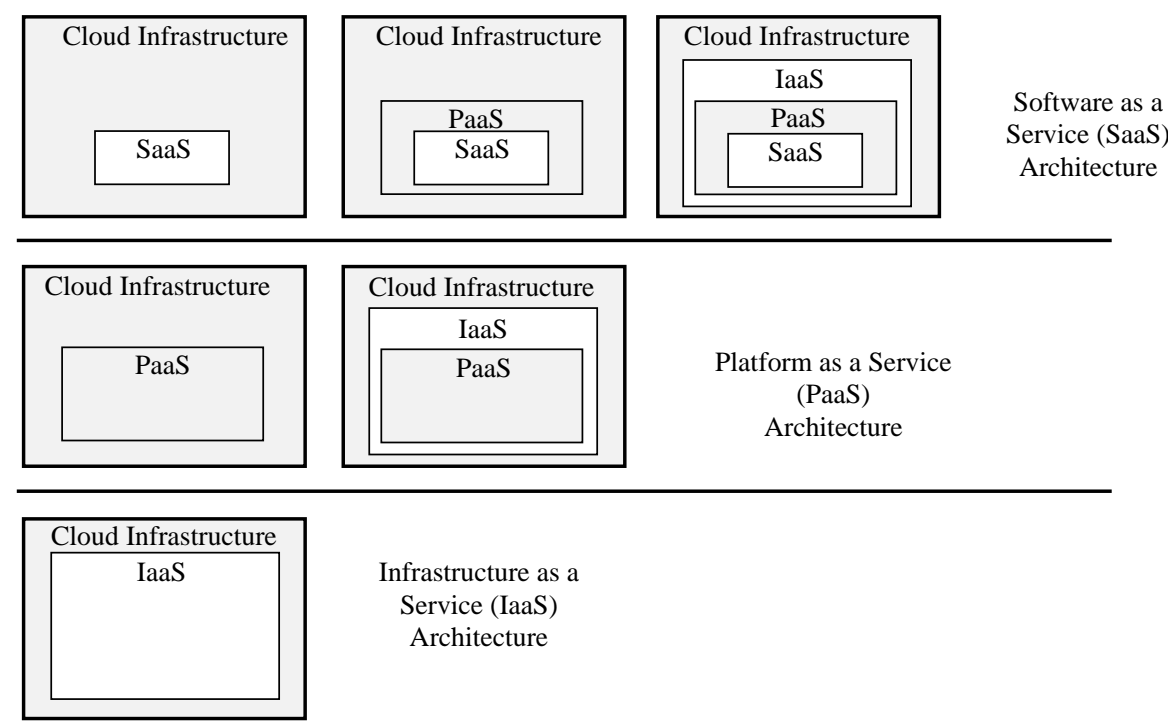

Fig.3. The Hierarchical view of cloud computing service models [20].

Fig. 3 depicts the hierarchical view of cloud computing service models. Based on this figure, in any of these service models, the customers have total control over the infrastructure's cloud providers. Between these three (3) service models, it can be noticed that IaaS is the service model that has the maximum control over the infrastructure providers. While compared to IaaS, PaaS has the minimum control over the infrastructure providers. All the services offered in IaaS, are part of the cloud providers' responsibilities. Lastly, SaaS is an infrastructure distributed to customers throughout a network. The customers of this service have very miniature control over the infrastructure. To manage and control the fundamental infrastructure and platform is the segment of the cloud providers' responsibilities.

\section{The Cloud Computing Deployment Models}

Choosing the suitable type of $\mathrm{CC}$ to be implemented by an institution is the first important step to take as it promises a successfully $\mathrm{CC}$ implementation by that institution as different types of $\mathrm{CC}$ require diverse skills and resource. According to Chauhan et al. [21], many institutions that have been failing in the implementation of their CC failed because of choosing the wrong CC. Institutions must examine their data precisely, before deciding which type of $\mathrm{CC}$ to choose so that they can avoid failure of implementation.

There exist four (4) of models that have been totally adopted in any $\mathrm{CC}$ research-based, according to their distribution and physical location. There based on previous researches on $\mathrm{CC}$, the deployment models of $\mathrm{CC}$ have been classified as the following.

\section{A. The private cloud}

This deployment model functioned especially on behalf of company meaning this type of CC services is not accessible by the public; it is survived by the company. It might exist on or off the locations as the users of this type of CC can be from diverse units or departments but belonging to the same specific company. The private cloud is known as the most security cloud as it data processes are controlled and managed in the company exclusive of any limitation of bandwidth network, security disclosures, and legitimate requirements using services of public cloud may necessitate [22]. In the private cloud, it possible that the fundamental cloud's infrastructure can be leased or owned, even operated and managed by the company itself, a third-party or both [5]. It delivers many outcomes to a public cloud computing environment, for example, becoming a service-based also elastic. Examples Amazon Virtual Private Cloud [4].

According to Parsi \& Laharika [23], private clouds are classified into two (2) variations which are:

\section{- The on premise private cloud}

Also, called as the internal cloud, this type of private cloud is held inside the institution's personal data centre. It offers an additional uniform procedure plus security, yet is frequently restricted in size and scalability. Moreover, an organization's Information Technology (IT) unit would encounter the costs of capital and operational for the physical resources with this model. This type of private cloud is best utilized for applications (apps) which 
necessitate total control and configurability of the different infrastructures and security (protection).

\section{- The externally-hosted private cloud}

The type of private cloud model called the externally hosted is held by an outside CC provider. This CC service provider encourages a restrictive $\mathrm{CC}$ environment with complete guarantees of confidentiality. This type of private clouds is advised to institutions that do not favour using a public cloud infrastructure due to the fact of the because of the risks associated with the physical resources sharing.

According to Thakur et al. [14], some characteristics of private cloud are as below:

\section{- Enhanced Security Measures}

In an IT sector security is one of the requirement that many institutions seek for particularly when it comes to financial institutions. For example, the security and confidentiality issues are the principal concern in the banks. The private cloud model arrives well furnished with a customizable and thorough firewall and a plethora of security and confidentiality tools that guarantee extreme safety against illegal usages, such as hacking and other.

\section{- Dedicated Resources}

One of the principles of private cloud is "no bargain". Like a supporter of private cloud, enterprises have their personal dedicated resources, for example, the time of processor and the data buses that guarantee ideal execution.

\section{- Better Customization}

The private cloud model is acquiescent and customizable as it can be built to outfit the precise requests of a business. This in turns allows the business to take additional control over their own data to ensure security.

The private cloud models are consenting and adaptable as they can be made to outfit the exact solicitations of a business. This in turns permits the business to have more control over their information keeping in mind the end goal to guarantee security.

\section{B. The public cloud}

The public cloud model comes with many features as it offers applications, data storages, and many different services to its users coming from its service provider. This is based on the characteristic of the pay-as-you-go model. This cloud model is built with a perspective to provide boundless memory storage and expanded data transmission through the Internet to all organizations. It is also hosted, owned, and operated by a third-party service provider. It is as well as takes into account every sort of prerequisites from little, medium and enormous organizations [24]. It is considerate as the easiest to be setup since it liberates that supporter from burdens of equipment, application or transfer speed costs. Organization pays for only those services and resources they have used. Customers must pay their bill of public cloud services, monthly.

It does not require any hardware device as it can function on the major principle of storage demand scalability. The accepted examples of public clouds are Amazon Elastic Cloud Compute, Google App Engine, and Blue Cloud by IBM and Azure Services Platform by Windows [22].

Briefly, this cloud is known for its availability to the public or bigger of the institution from the third party that is based on providing services to its client through the internet. Nevertheless, it does not mean that the client's data will be publically exposed to be visible as the public cloud dealers always deliver an authorized and authentication access control for the clients. This cloud provides a cost-effective and elastics meaning to solutions deployed [22].

According to Parsi \& Laharika [23] public cloud provides four (4) basic characteristics, which are the following:

\section{Flexible and Elastic Environment}

The public cloud for example Google App engine and Amazon elastic CC provides to its customers a greatly adaptable environment of the cloud. It empowers customers in sharing and storing information based on the customers' own abilities. The CC owner also can choose what they want to share and what they do not want to share with their customers.

\section{Freedom of Self-Service}

The public cloud inspires it customers in making a cloud all alone exclusive of taking anybody's assistance. This is called as the pre-configured clouds, which exist on the Internet. The principal thing is that organizations that desire to choose the public cloud need to do is to visit the portals of the public cloud begin with it. They do not need to have relied on any third-party support in making or running this sort of cloud. As it will directly be overseen and took care of by them like they will be the principal owner of it.

\section{Pay for what is used}

This specific characteristic empowers the technology of cloud to be extra accessible by organisations to operate in a synchronized manner. The further organization uses the services of cloud, the well prosperous the future business will be. Nevertheless, the charging for the payment is done based on the basic cloud services utilised by customers.

\section{Availability and Reliability}

The fact that the public cloud is accessible to all and believes in agility is one of the many other characteristics if the public cloud. The users have the possibility to time their work at whatever period they want also from whatever side of the globe. Not just customers end up being free to run basic assignments of the business but they are additionally extra productive in reinforcing customer relationships over the globe. 


\section{The hybrid cloud}

This cloud is made of many of both private or public cloud which is shared between the different institutions that have similar interests and requirements, it's can be internally managed and it can also be managed by the third-party which is inside or outside hosted. The bills of this cloud are increasing over some clients; consequently, some of the bill savings benefits of CC are accomplished. This could assist in limiting the benefits spending expenses for its founding as the prices are distributed among the companies; most of the government agencies in a single region cloud may be shareable, but not the non-government agencies [23]. However, organizations can maintain their cost and security at a reasonable level; but at the same time, there are some issues regarding standardization and interoperability of clouds, which should be considered [24].

According to Sujay [25], some characteristics of the hybrid clouds are:

1. Optimal use: The typical centres of data in the server resources are used from five (5) to twenty (20) \%. The reason behind that is the crest loads, which are ten (10) times higher than that of the typical burden. In this way, servers are generally sitting still - making pointless costs. Hybrid cloud could extend server use by scaling out to open assets to take care of hosts.

2. Data centre consolidation: Rather than giving the capacity to adjust to most sceptical situation circumstances, a private cloud simply needs resources in typical cases. The contrasting option to impact out grants server union and therefore achieving the abatement in working costs. This incorporates the hardware, power, cooling, maintaining, as well as service costs.
3. Risk transfer: Organizations personally are maintaining and running their server (the centre of their data) and private cloud. The service provider of the public cloud provider musts ensures an extreme uptime for their service. Utilizing the hybrid cloud, the danger of misestimating workload is relocated to the cloud seller from the service operator. The clear majority of the cloud providers have the SLAs, which guarantee an uptime of more than $99.9 \%$ consistently, for example, downtime of max. Nine (9) hours for each year.

4. Availability: The extreme accessibility in the corporate server (the centre of their data) is troublesome as well as costly, as it necessitates data redundancy, data reinforcements, and geographical scattering. Particularly within the organizations where Information Technology is not $\mathrm{t}$ the focus corporate, the skill around there is somewhat restricted. In a hybrid cloud, the public cloud might scale up or completely overtake operations if the organization's server (the centre of their data) is not available because of some failures and some attacks of Distributed Denial of Service (DDoS).

\section{Community Cloud}

The community cloud infrastructure is supervised, then utilized by a different number of institutions that have the same core business, projects or shareable demands infrastructures such as software and hardware so that the running costs of IT can be reduced. Therefore, this cloud can be manageable by either the joined institutions or the cloud that provides the services [14]. Academic clouds are an example of community cloud.

The cloud computing deployment models are graphically depicted in Fig. 4.

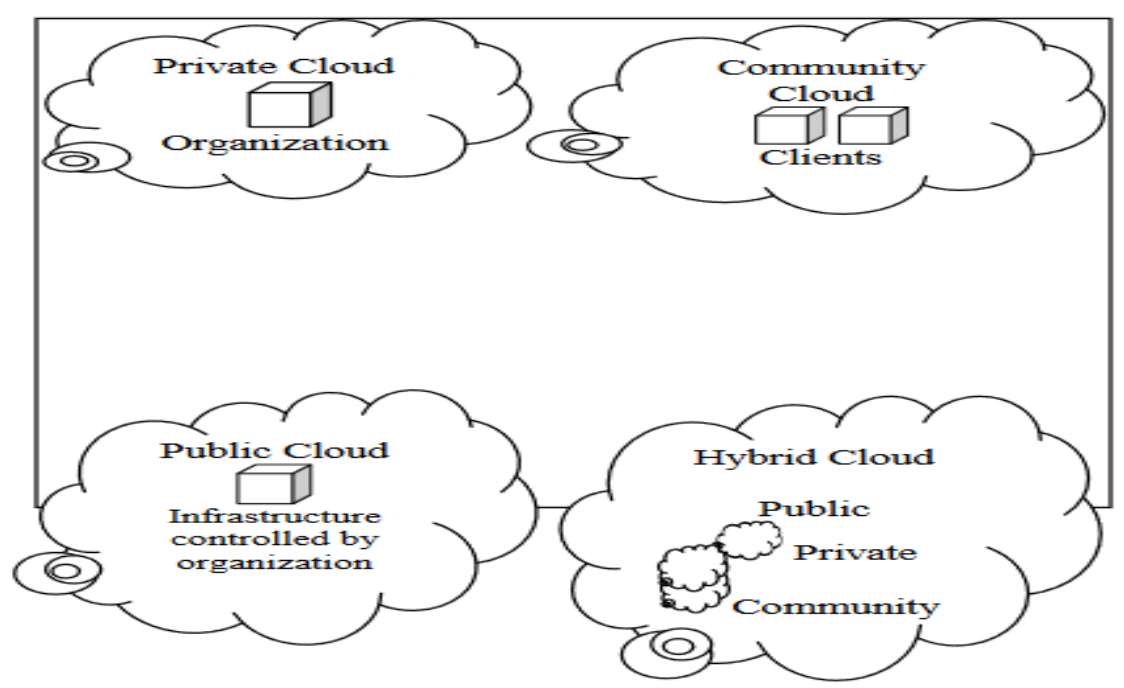

Fig.4. The cloud computing deployment models [14].

\section{The RoOTs OF CLOUd COMPUTING}

$\mathrm{CC}$ originality is to be followed by the evolution of countless technologies innovations most strikingly the advancement of hardware technology for example multi- core chips, virtualization, and managing systems, for example, automation of data centre, internet technologies advancement like Web services, service- oriented architecture, Web 2.0, distributed computing notion, grid computing as well as cluster computing [26]. 


\section{A. The grid computing}

Begun during the mid-1990s, like a consequence of the requirement for the computing systems obliging the next expanding requirement for very quick calculating scientific intensive data applications. The fundamental objective of the grid is uniting huge computer that is distributed, capacity assets as well as interface remotely found PCs through an extensive system, in this way wiping out the topographical barriers and guaranteeing that unmoving resources are used to the best [27]. Therefore, Grid Computing incorporate numerous managerial fields from various topographical localities to resolve a solitary errand and are rapidly released [28]. The standard protocols expansion from different Grid Computing activities provided the transportation of resources of computing throughout the Internet ondemand [26]. Nevertheless, the acknowledgment of the quality of service $(\mathrm{QoS})$ in the grids is the significant test. Grid contrasts from the cluster as in network resources are intended to be slackly paired. The inevitable developments in computing carry about the necessity for the release of computing-as-a-service, quite than computing-as-a-product. As indicated by Hashemi \& Bardsiri [29], they explained Grid Computing in terms of application usage, data and storage as well as network resource saying "Grid computing is categorically defined or explained in terms of distributed computing which involves a large amount of coordination also sharing computing, application, data storage among other, as well as network resources across dispersed organization.” The organization must be dynamically and geographically dispersed for it the real essence of grid computing to be clearer and more meaningful to the organization concerned. Further explanation showed that the reason and vision behind grid computing were to allow access to computer-based resources. It also has the following characteristics:

- Large Scale: The capability of dealing with the huge quantity of resources, which could be a bit costly as the cost, can be few millions.

- Geographical Distribution: the ability to access the resource from distant places.

- Heterogeneity: the ability to host both software and hardware that can range from data to files, software component, and even programs.

- Resource sharing: allowing access of resources in a grid belonging different organizations.

- Multiple Administrations: the ability for different organisations to create distinctive security as well as policies of admin so that the resources they own will be accessible and usable.

- Dependable Access: the ability of the grid computing to ensure safe delivery of service underneath established service quality.

- Consistent Access: the ability of the grid to be created through standard services, protocols as well as the interface to interact.

\section{B. Hardware Virtualization}

The virtualization idea get is originality from the time virtual machines were introduced (an occurrence of the physical machines) by IBM during the 1960s [30]. The thought behind utilizing virtual machines (VM) is because they empower computing resource-sharing (hardware) as well as time. Therefore, a virtual machines sponsor advance decrease of equipment like hardware expense but then enhancing profitability by permitting different clients synchronous access to the instance of a computing resource [28]. The hardware virtualization offers to the clients the capacity of running different software on a similar physical machine, hiding all features that are detailed in the physical machine from the clients.

\section{Autonomic Computing}

According to Boom [31], autonomic computing combines both the study and capability of the computer system with the ability to achieve autonomously desired behaviour, further explanation was using a specific example saying "self-tuned system has the ability to tune their respective performance based on the needs of their intended missions. In this case, the self-protected system automatically handles intrusion attacks from an external source, as a self-manage system do not really requirement human-made configuration. Another distinct behaviour is that self-healing system is capable of repairing them as the case maybe while the self-managed system can also be constructed broadly as having the capability of managing all type of behaviour". According to Autonomic computing is described by four (4) important features which are self-optimization, self-protection, selfconfiguration, lastly self-healing [26].

\section{Web Services and Service-Oriented Architecture} (SOA)

Web Services (WS) open standard development have specially added to the integrated business systems enhancements and supporting. These innovations in Web services empower data sharing amongst running application upon various chatting platforms, in this way creating single internal data application's accessible by others throughout the internet [26]. Web services were developed throughout current renowned technologies such as Extensible Mark-up Language (XML) as well as Hypertext Transfer Protocol (HTTP), which are subsequently skilled on behalf of procurement of mechanisms to carry services and implement SOA.

SOA intended to address prerequisite of slackly paired, standard-based, as well as independent protocol dispersed computing [28]. The advancement of Web services empowers the creation of influential services supporting simple and quick access on-demand in a reliable manner.

Supercomputers have assumed the main part for calculation-intensive purposes, for example, quantum physics and climate conjecture applications, nevertheless, deploying supercomputers to do such errands is not practical, therefore, the advancement of cluster computing [28]. Cluster computing comprises of a 
gathering of parallel and distributed PCs working firmly together to perform an errand that would typically not be accomplished with a solitary PC. Cluster computing is generally connected over a quick Local Area Network (LAN) Internet [26]. The fundamentally favourable position of groups over single PC is the procurement for high accessibility, the load-adjusting and diminished expense of sending contrasted with conveying a supercomputer Internet [26]. High accessibility of cluster computing group is accomplished using repetitive hubs such that the hubs can provide for service in case of a failure of the system. Fig. 5 illustrates the convergence of these technologies and the development of cloud computing.

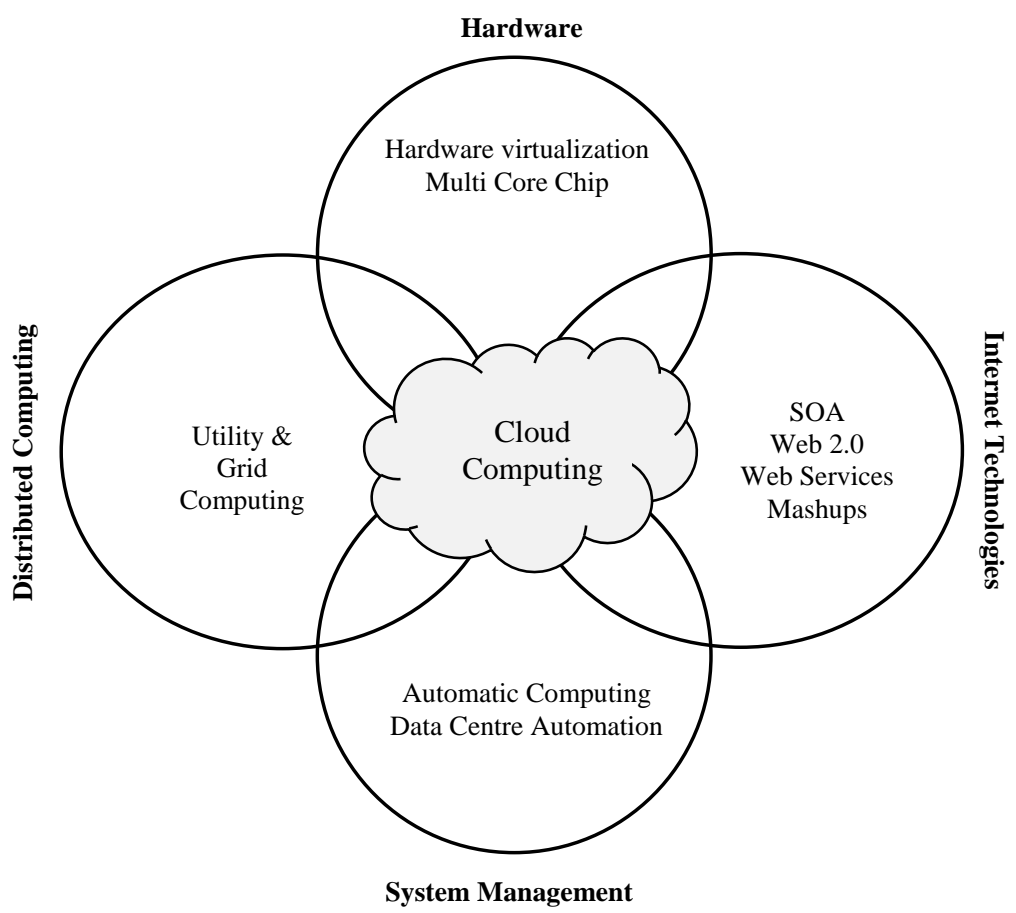

Fig. 5. The emergence of Cloud computing from the advancement in computing technologies [28].

\section{CONCLUSION}

Considering the historic growth of providing IT resources, cloud computing has been recognised as the freshest and most flexible delivery model of providing IT. It can be considered as the resulting evolution of the traditional on premise computing spanning outsourcing stages from aggregate to the specific, and from the multiseller outsourcing to an advantage free delivery. Cloud computing is a technology used for increasing the capacity or add capabilities progressively without putting resources in new infrastructure, training new personnel, or licensing new software. It is a very promising technology, which is allowing organisations to effectively manage their resource limitations with slightest amount of capital investment and meet dynamic demands efficiently. Cloud computing offers deployment architecture, with the capability to address vulnerabilities recognised in traditional IS yet its dynamic qualities can deflect the effectiveness of traditional countermeasures. Different cloud models can be selected varying upon the specific desires of the organisation. This paper discussed the concept of cloud by explaining it evolution and history, and giving different definitions of cloud computing. It also addressed the service and deployment models of cloud computing, it characteristics and root.

\section{REFERENCES}

[1] Akande, A.O., N.A. April, and J.-P. Van Belle. Management Issues with Cloud Computing. in Proceedings of the Second International Conference on Innovative Computing and Cloud Computing. 2013. ACM.

[2] Sharma, R. and R.K. Trivedi, Literature review: Cloud Computing-Security Issues, Solution and Technologies. International Journal of Engineering Research ISSN, 2013: p. 2319-6890.

[3] Khan, A.W., S.U. Khan, M. Ilyas, and M.I. Azeem, A literature survey on data privacy/protection issues and challenges in cloud computing. IOSR Journal of Computer Engineering (IOSRJCE) ISSN, 2012: p. 22780661 .

[4] Sriram, I. and A. Khajeh-Hosseini, Research agenda in cloud technologies. arXiv preprint arXiv:1001.3259, 2010

[5] Mell, P. and T. Grance, The NIST definition of cloud computing. 2011.

[6] Islam, S. and J.-C. Grégoire, Giving users an edge: A flexible Cloud model and its application for multimedia. Future Generation Computer Systems, 2012. 28(6): p. 823-832.

[7] Böhm, M., S. Leimeister, C. Riedl, and H. Krcmar, Cloud computing and computing evolution. B. Smith,"An approach to graphs of linear forms (Unpublished work style)," unpublished, 2010.

[8] Stavinoha, K.E., What is Cloud Computing and Why Do We Need It. 2010, Citeseer. 
[9] Rai, R., G. Sahoo, and S. Mehfuz, Securing software as a service model of cloud computing: Issues and solutions. arXiv preprint arXiv:1309.2426, 2013.

[10] Khorshed, M.T., A.S. Ali, and S.A. Wasimi, A survey on gaps, threat remediation challenges and some thoughts for proactive attack detection in cloud computing. Future Generation computer systems, 2012. 28(6): p. 833-851.

[11] Oliveira, T., M. Thomas, and M. Espadanal, Assessing the determinants of cloud computing adoption: An analysis of the manufacturing and services sectors. Information \& Management, 2014. 51(5): p. 497-510.

[12] Dillon, T., C. Wu, and E. Chang. Cloud computing: issues and challenges. in 2010 24th IEEE international conference on advanced information networking and applications. 2010. Ieee.

[13] Srinivas, J., K.V.S. Reddy, and A.M. Qyser, Cloud computing basics. International journal of advanced research in computer and communication engineering, 2012. 1(5).

[14] Thakur, N., D. Bisen, V. Rohit, and N. Gupta, Review on Cloud Computing: Issues, Services and Models. International Journal of Computer Applications, 2014. 91(9).

[15] Ashrafa, I., An Overview of Service Models of Cloud Computing. Int. J. of Multidisciplinary and Current research, 2014.

[16] Rao, C.C., M. Leelarani, and Y.R. Kumar, Cloud: Computing Services and Deployment Models. International Journal of Engineering and Computer Science, 2013. 2(12).

[17] Khurana, S. and A.G. Verma, Comparison of Cloud Computing Service Models: SaaS, PaaS, IaaS. International Journal of Electronics \& Communication Technology IJECT, 2013. 4.

[18] Fernandes, D.A., L.F. Soares, J.V. Gomes, M.M. Freire, and P.R. Inácio, Security issues in cloud environments: a survey. International Journal of Information Security, 2014. 13(2): p. 113-170.

[19] Salleh, S.M., S.Y. Teoh, and C. Chan. Cloud Enterprise Systems: A Review Of Literature And Its Adoption. in PACIS. 2012.

[20] Tehrani, S.R. and F. Shirazi. Factors influencing the adoption of cloud computing by small and medium size enterprises (SMEs). in International Conference on Human Interface and the Management of Information. 2014. Springer.

[21] Chauhan, V.K., K. Bansal, and P. Alappanavar, Exposing cloud computing as a failure. International journal of engineering science and technology, 2012. 4(4).

[22] Kim, W., Cloud Computing: Today and Tomorrow. Journal of object technology, 2009. 8(1): p. 65-72.

[23] Parsi, K. and M. Laharika, A Comparative Study of Different Deployment Models in a Cloud. International Journal of Advanced Research in Computer Science and Software Engineering, 2013. 3(5): p. 512-515.

[24] Grossman, R.L., The case for cloud computing. IT professional, 2009. 11(2): p. 23-27.

[25] Sujay, R., Hybrid cloud: A new era. International Journal of Computer Science and Technology (IJCST), 2011. 2(2): p. 323-326.

[26] Badger, L., T. Grance, R. Patt-Corner, and J. Voas, Draft cloud computing synopsis and recommendations. NIST special publication, 2011. 800: p. 146.

[27] Sadashiv, N. and S.D. Kumar. Cluster, grid and cloud computing: A detailed comparison. in Computer Science \& Education (ICCSE), 2011 6th International Conference on. 2011. IEEE.
[28] Muhammad, A.R., Towards cloud adoption in Africa: The case of Nigeria. International Journal of Scientific \& Engineering Research, 6 (1), 2015: p. 657-664.

[29] Hashemi, S.M. and A.K. Bardsiri, Cloud computing Vs. grid computing. ARPN Journal of Systems and Software, 2012. 2(5): p. 188-194.

[30] Voorsluys, W., J. Broberg, and R. Buyya, Introduction to cloud computing. Cloud computing: Principles and paradigms, 2011: p. 1-44.

[31] Boon, M. What is autonomic computing? 2011.

\section{Authors' Profiles}

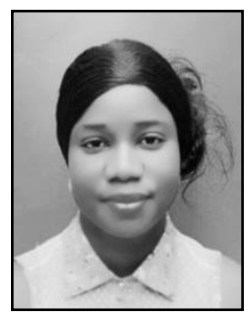

Tinankoria Diaby received her BSc (Hons) in Information Technology (Business in Information System) in 2014 from Asia Pacific University of Technology and Innovation (APU), Kuala Lumpur Malaysia; and currently, enrolling her MSc in Information Technology (IT Management) from Asia Pacific University of Technology and Innovation (APU), Kuala Lumpur Malaysia; Her main research interest covers a broad range of various areas in Information Technology, especially in the areas of Information Technology Management related to business environments.

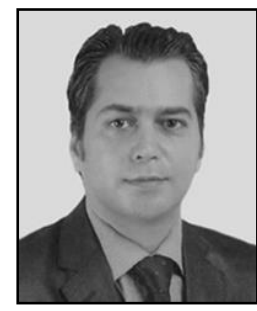

Babak Bashari Rad received his B.Sc. of computer engineering (software) in 1996 and M.Sc. of computer engineering (Artificial intelligence and robotics) in 2001 from University of Shiraz; and Ph.D of computer science (information security) in 2013 from University technology of Malaysia. Currently, he is the program leader of post graduate studies and senior lecturer in the school of computing, Asia Pacific University of Technology and Innovation (APU), Kuala Lumpur Malaysia. His main research interest covers a broad range of various areas in computer science and information technology including information security, malware detection, machine learning, artificial intelligence, image processing, robotics, and other relevant fields.

How to cite this paper: Tinankoria Diaby, Babak Bashari Rad,"Cloud Computing: A review of the Concepts and Deployment Models", International Journal of Information Technology and Computer Science(IJITCS), Vol.9, No.6, pp.50-58, 2017. DOI: 10.5815/ijitcs.2017.06.07 